\section{PO 8411 MOLECULAR CHARACTERISATION OF GP41 AND GP120 V3 LOOP IN HIV-1C PATIENTS FAILING SALVAGE THERAPY IN BOTSWANA}

${ }^{1}$ Nokuthula S Ndlovu*, ${ }^{2}$ Kaelo Seatla. 'Midlands State University, Gweru, Zimbabwe, Botswana Harvard Partnership; ${ }^{2}$ Botswana Harvard Partnership, University of Botswana, Gaborone, Botswana

\subsection{6/bmjgh-2019-EDC.87}

Background Triple class drug-resistant HIV-1 infection remains a global challenge in individuals with extensive antiretroviral treatment (ART) experience, in terms of high mortality and probability of onward transmission. New therapeutic options within old and new drug classes are therefore essential. We determined if patients failing salvage therapy in Botswana are eligible for maraviroc (MVC) and enfuvirtide (T20) viral entry inhibitors based on the coreceptor usage and drug-resistant mutations in envelope gp120 and gp41.

Methods A total of 38 deep salvage patients were included in the analysis. We amplified and sequenced gp41 and V3 regions of HIV-1 envelope. Drug resistance mutations were analysed according to the IAS-USA 2017 reference mutation lists. Coreceptor usage was determined using PSSM and Geno2Pheno using a false-positive rate (FPR) of $10 \%$.

Results Among 38 participants, 34 (89\%) were successfully sequenced and amplified gp41 and 26 (68\%) gp120 V3 loop sequences were obtained. Major T20 mutation G36S was obtained in $1 / 34$ samples $(5.8 \%)$ within the study population. Polymorphisms I169V(97\%), I135L(100\%), E151A(70.6\%) and N42S(70.6\%) were detected in HR1 and HR2 of gp41. CXCR4 coreceptor associated use, mutation L34M in gp41 HR1 was detected in 2 samples (5\%). Analysis of coreceptor usage showed (17/26) $65.4 \%$ use of CCR5, and a (9/26) $34.6 \%$ use of the CXCR4 coreceptor.

Conclusion A moderately high proportion of treatment-experienced (deep salvage) participants had CXCR4 coreceptor using strains. The use of maraviroc in Botswana would require coreceptor tropism testing. Non-T20 treatment experience in Botswana reduces the prevalence of the major mutations that confer resistance to the drug. T20 is therefore a potential alternative drug for patients failing salvage therapy in Botswana.

\section{PO 8414 EVALUATION OF MYCOBACTERIUM TUBERCULOSIS COMPLEX (MTBC) CULTURE METHODS IN MYCOBACTERIUM AFRICANUM-ENDEMIC REGION OF WEST AFRICA}

${ }^{1}$ Alieu K Faburay*, ${ }^{1}$ Francis S Mendy, 'Sarjo A Gibba, ${ }^{1}$ Modou Lamin, ${ }^{1}$ Basil Sambou, ${ }^{1}$ Alieu Mendy, ${ }^{1}$ Tutty I Faal-Jawara, ${ }^{2}$ Florian Gehre, ${ }^{1}$ Jayne Sutherland, ${ }^{1}$ Beate Kampmann, 'Ousman Secka. 'Medical Research Council Unit The Gambia at LSHTM, The Gambia; ${ }^{2}$ Bernhard-Nocht-Institute for Tropical Medicine, Hamburg, Germany

\subsection{6/bmjgh-2019-EDC.88}

Background With the endemic Mycobacterium africanum (Maf), West African laboratories use glycerol and pyruvate in separate LJ cultures (LJG and LJP) for isolation of MTBC. The aim of this work is to evaluate if combining both glycerol and pyruvate in a single LJ medium (LJGP) will lead to comparable growth characteristics and time to detection in comparison to LJG, LJP and MGIT 960.

Method Total of 118 smear-positive sputum samples were processed using 4\% NaOH-NALC decontamination method.
The decontaminated samples were inoculated parallel on LJG, LGP, MGIT 960 and LJGP. Positive cultures were confirmed using Ziehl-Neelsen staining method. MTBC identification was done using the Capilia TBNeo kit and spoligotyping used for speciation.

Results The recovery rate for LJG, LJP, LJPG and MGIT was found to be $73.7 \%$ (87/118), 82.2\% (96/118), 83.9\% (99/118) and $93.2 \%(110 / 118)$ respectively. No significant agreement was observed between the LJPG and MGIT 960 with Kappa values of -0.105 ( $p$-value $=0.199)$. However, there was significant agreement between LJGP and LJG and LJP with Kappa value of 0736 (p-value $<0.001$ ) and 0.756 (p-value $<0.001$ ), respectively. There were 70 Euro-American, 34 Maf, 9 East-Asian, 2 Indo-Oceanic, 2 East-African-Indian and $1 \mathrm{M}$. Bovis. LJGP have better Maf recovery rate, $85.3 \%$ (29/34) in comparison to MGIT 960, 79.4\% (27/34), LJP, 76.5\% (26/34) and LJG, $61.8 \%$ (21/34). Seven of the 8 MGIT negatives that were LJPG positive were $M$. africanum and $1 \mathrm{M}$. bovis.

Conclusion LJPG has a better detection rate and time to positivity compared to LJG and LJP and was shown to have a better Maf recovery than other LJ methods and MGIT 960. It is evident that LJGP is a promising culture tool for Mafendemic West African countries that will not only increase MTBC recovery rate in combination with MGIT, but also leads to better detection of Maf.

\section{PO 8417 RISING TRENDS IN TB MORTALITY AMID DECLINE IN CASES NOTIFIED IN A RURAL COUNTY IN KENYA: COHORT STUDY}

${ }^{2}$ Moses Ngari*, ${ }^{1}$ Osman Abdullahi, ${ }^{3}$ Deche Sanga, ${ }^{4}$ Geoffrey Katana, ${ }^{1}$ Annie Willetts. ${ }^{1}$ Pwani University, Department of Public Health, Kilifi, Kenya; ${ }^{2}$ KEMRI Wellcome Trust Research Programme, Kilifi; ${ }^{3}$ Kilifi County TB Control Programme, Kilifi, Kenya; ${ }^{4}$ Kilifi County Department of Public Health, Kilifi, Kenya

\subsection{6/bmjgh-2019-EDC.89}

Background Despite introduction of rapid and accurate diagnostic tools and aggressive treatment for tuberculosis (TB), it is still a global health problem. In 2016, globally, 1.7 million people died of TB, 95\% from resource-poor countries. This study aimed to estimate changing trends in all-cause mortality rate and identify features associated with mortality among suspected TB patients on treatment.

Methods A cohort study of patients registered in a TB surveillance system from 2012 to 2016 and followed up for six months during TB treatment. The outcome was all-cause mortality within six months of TB treatment. The exposures examined were demographic and clinical features at the time of starting TB treatment.

Results A total of 10,717 participants, median (IQR) age 33 (24-45) years, of which 3163 (30\%) were HIV-infected were included in the analyses. During follow-up of 5175.5 personyears (PY), 585/10,717 (5.5\%) participants died; mortality rate was 12.2 (95\% CI $11.3,13.3)$ deaths per $100 \mathrm{PY}$. The yearly mortality rate increased from 7.79 (95\% CI 6.35, 9.54) in 2012 to $17.73(95 \%$ CI $14.93,21.06)$ in 2016 per $100 \mathrm{PY}$ $\left(\mathrm{P}_{\text {trend }}<0.001\right)$ but the number of suspected-TB notifications declined from 2610 (24\%) in 2012 to 1689 (16\%) in 2016 $\left(\mathrm{P}_{\text {trend }}=0.02\right) .77 \%$ of all deaths occurred by month three. Mortality among HIV-infected participants was higher (325/ $3163 ; 10.3 \%)$ than among HIV-non-infected participants $(251 /$ $7413 ; 3.4 \% ; \mathrm{p}<0.001)$. Old age, being a female, type of TB diagnosis used, body mass index $(\mathrm{BMI})<18.5$, HIV status and 
year of diagnosis were associated with mortality in the multivariate regression model.

Conclusion This large population level TB study identifies an alarming trend of patients dying within months of starting treatment. These early deaths could be due to late diagnosis and multidrug-resistance. The study warrants further investigation to go beyond already established indicators which remained constant (including HIV co-infection), to explore host, disease or health system related factors that may explain the observed trend.

\section{PO 8418 NEW MALARIA EPIDEMIOLOGY IN COASTAL LAGOON OF BENIN: PLASMODIUM INFECTION IN ANOPHELES MELAS}

Claudiane Adigbonon*, Benoît S Assogba, Luc S Djogbenou. University of Abomey-Calavi, Cotonou, Benin

\subsection{6/bmjgh-2019-EDC.90}

Introduction Malaria is a worldwide disease affecting many people particularly in the tropical and sub-tropical areas. It is caused by Plasmodium parasites and essentially transmitted by female mosquitoes belonging to the Anopheles genus. Our understanding of the infectivity of these vectors to Plasmodium is necessary to design sustainable strategies for their control. This aspect remains unknown in the coastal and lagoon area of Benin where Anopheles melas and Anopheles coluzzii are sympatric. This study aims to investigate the infectivity of these two vectors to Plasmodium to understand their role in malaria transmission in southern Benin.

Methods Insecticide spray catch technique was used to collect females in 80 houses randomly selected in our study site. Three hundred and twenty (320) females were identified using PCR-species technique, Plasmodium infection was determined by the TaqMan method during the dry season. This assay detects all four malaria-causing species and discriminates Plasmodium falciparum from Plasmodium ovale, Plasmodium vivax and Plasmodium malariae (OVM).

Results During the dry season, the sporozoïte rates were $0.2 \%$ and $0.3 \%$ for Anopheles melas and Anopheles coluzzii, respectively. However, we observed that positivity to the OVM (one of Plasmodium ovale, Plasmodium vivax and Plasmodium malariae species) was significantly higher in Anopheles melas (95\%) than in Anopheles coluzzii (33.33\%) (Chi-sq=15 857, $\mathrm{df}=1, \mathrm{p}<0.001)$. These results indicated that Anopheles melas is more infected by one of the species Plasmodium ovale, Plamodium vivax and Plasmodium malariae than by Plasmodium falciparum, contrarily to Anopheles coluzzii.

Conclusion These findings reinforce the debate on the role of Anopheles melas in malaria transmission in coastal lagoon areas of Benin.

\section{PO 8419 SPATIO-TEMPORAL MAPPING OF ASYMPTOMATIC AND CLINICAL MALARIA INFECTIONS REVEALS FOCI OF MALARIA TRANSMISSION FOR TARGETED CONTROL INTERVENTIONS}

\footnotetext{
${ }^{1}$ Makhtar Niang* ${ }^{2}$ Cheikh Talla, ${ }^{3}$ Nafissatou Diagne, ${ }^{4}$ Fatoutama Diene-Sarr, ${ }^{3}$ Cheikh Sokhna. ${ }^{1}$ Immunology Unit, Institut Pasteur de Dakar, Senegal; ${ }^{2}$ Epidemiology Unit, Institut Pasteur of Dakar, Senegal; ${ }^{3}$ Institute for Research and Development, Dakar, Senegal; ${ }^{4}$ Epidemiology Unit, Institut Pasteur de Dakar, Senegal
}

10.1136/bmjgh-2019-EDC.91
Background The global decline of malaria incidence over the past decade has led to the thought that elimination could be achieved. This has resulted in an increased interest to design strategies to target the hidden reservoir of asymptomatic infections among populations and interrupt on-going residual local malaria transmission. This study explored the reservoir of asymptomatic Plasmodium infections and its relationship with subsequent clinical malaria infections in low-transmission areas in Senegal.

Methods Cross-sectional surveys were carried out in 2013, 2014, 2015, and 2016 and combined with longitudinal follow-up to determine and geolocalise both asymptomatic and clinical malaria episodes in Dielmo and Ndiop, Senegal. The prevalence of asymptomatic Plasmodium carriage in the community was investigated by real-time PCR while clinical malaria attacks were identified at health facilities during the transmission season. All households were georeferenced to spatially map asymptomatic and clinical infections.

Results The study revealed substantial asymptomatic infections with average parasite carriage of $8.11 \%$ and $7 \%$ in Dielmo and Ndiop, respectively. P. falciparum accounted for most asymptomatic infections (more than 90\%). In Dielmo, 95\% of asymptomatic infections clustered within the same geographical areas while infections were disparate in Ndiop. Preliminary fine-scale mapping of asymptomatic and clinical malaria infections identified clusters of higher malaria incidence interpreted as foci of transmission across the four-year study period with 95\%-98\% of clinical infections occurring in households where an asymptomatic malaria infection existed.

Conclusion This study revealed substantial asymptomatic Plasmodium infections in both settings throughout the four-year study period and spatial clusters of malaria infections at the microepidemiological level. Together, these findings could offer a feasible approach for a rational targeting of malaria control interventions to achieve elimination.

\section{PO 8421 LITERATURE REVIEW OF BIOMARKERS FOR HUMAN AFRICAN TRYPANOSOMIASIS POST-TREATMENT FOLLOW-UP}

${ }^{1}$ Lukusa Ngay*, ${ }^{2}$ Veerle Lejon, ${ }^{1}$ Mumba Ngoyi. ${ }^{1}$ Institut National de Recherche Biomedicale (INRB), Kinshasa, Democratic Republic of the Congo; ${ }^{2}$ Institut de Recherche pour le developpement (IRD)/Montpellier, France

\subsection{6/bmjgh-2019-EDC.92}

Introduction Human African trypanosomiasis (HAT) is caused by Trypanosoma brucei gambiense and rhodesiense and is transmitted to humans by tsetse flies in sub-Saharan Africa. To detect cure or treatment failure, patients are followed up after treatment integrating the use of biomarkers in blood or cerebrospinal fluid (CSF).

Methods A systematic review of the literature according to the PRISMA Statement for Reporting Systematic Reviews was done, focusing on biological markers for HAT post-treatment follow-up. Articles were retrieved from PubMed (https://www. ncbi.nlm.nih.gov/pubmed/) by using keywords: Human African Trypanosomiasis, Biomarkers, Follow up, Post treatment.

Results A panel of biomarkers is used to detect relapses or to confirm recovery. For post-treatment follow-up, an examination of the CSF is performed. White blood cell counts in CSF with a defined cut-off value have been proven to be the most accurate to assess the treatment outcome. The intrathecal immunoglobulin $M$ synthesis is a specific and sensitive parameter for 